\title{
DISCRIMINATIVE OUTPUT CODING FEATURES FOR SPEECH RECOGNITION
}

\author{
Omid Dehzangi ${ }^{1}$, Bin Ma ${ }^{2}$, Eng Siong Chng ${ }^{1}$, Haizhou Li ${ }^{1,2}$ \\ ${ }^{1}$ School of Computer Engineering, Nanyang Technological University, Singapore \\ ${ }^{2}$ Institute for Infocomm Research, Singapore \\ \{Omid0002, aseschng\}@ntu.edu.sg, \{mabin, hli\}@i2r.a-star.edu.sg
}

\begin{abstract}
This paper presents a novel approach of discriminative acoustic feature extraction for speech recognition using output coding technique. A high dimensional feature space for higher discriminative capability is constructed by expanding MFCC coefficients with polynomial expansion. In order to fit the discriminative features in the hidden Markov model structure of speech recognition, the high dimensional feature vectors are further projected into a low dimensional feature space using the output scores of a set of SVMs. Each of the SVMs is trained in one phone versus the rest manner so that each of the resulting feature dimensions can provide effective information to differ one phone from the others. The discriminative features have been evaluated in the speech recognition task of the TIMIT corpus, and $72.18 \%$ phone accuracy has been achieved.
\end{abstract}

Index Terms - speech recognition, discriminative features, output coding, polynomial expansion, SVM

\section{INTRODUCTION}

A typical acoustic modeling approach for automatic speech recognition (ASR) system is to use the shorttime spectral features, such as mel-frequency cepstrum coefficients (MFCCs), as the basic acoustic feature vectors, and use a set of continuous density Gaussian mixture based hidden Markov models (HMMs) as the acoustic models. Model parameters in HMM-based speech recognition are normally estimated using the maximum likelihood (ML) criterion. In the past decade, extensive study has been made for the discriminative acoustic modeling methods.

As an alternative to the ML estimation, discriminative training of acoustic modeling, which aims to minimize the errors in training data, has been proved to be an effective method to improve the ASR performance. The maximum mutual information (MMI) $[1,2]$, minimum classification error (MCE) [3, 4], and minimum phone error (MPE) [5] have been successfully deployed in ASR. Recently the concept of large margin has been adopted in the discriminative training of acoustic models of ASR system [6, 7].

Compared with the model level discriminative training, there are few studies on the discriminative acoustic features. As the input to ASR systems, conventional acoustic feature vectors, which carry spectral information of the speech signal, are not discriminative in nature. In recent years, there has been much research interest in using linear/Nonlinear transformation on acoustic features and feeding the new features to train HMM models. For instance, hybrid connectionist-HMM systems [8] use discriminativelytrained neural networks to estimate the probability distribution among subword units given the acoustic observations and substitute the original features with these posterior probabilities. In another effort, TANDEM connectionist feature extraction [9] combines neural-net discriminative feature processing with Gaussian-mixture distribution modeling. As a linear approach, Linear Discriminant Analysis (LDA) has been used in ASR systems [10] attempting to find such basis vectors that the linear class separability is maximized.

In this paper, we would like to propose a new solution to extract the discriminative acoustic features for speech recognition. A high dimensional feature space, which is made by expanding the MFCC coefficients with polynomial expansion, will be constructed to improve their discriminative capabilities. In order to fit the discriminative features in the hidden Markov model structure of the state-of-the-art speech recognition system, the high dimensional feature vectors are further projected into a low dimensional feature space using the output scores of a set of SVMs. Each of the SVMs is trained independently in one phone versus the rest manner so that each of the resulting feature dimensions can provide effective information to differ one phone from the others. It is to make use of the output code feature space instead of the high dimensional feature space so that the discriminative features are applied for HMM modeling in place of MFCC features. We evaluate the proposed discriminative features in the speech recognition task of the TIMIT corpus. The resulting ASR system performs 
better than conventional MFCC based HMM baseline in phone recognition accuracy.

The rest of this paper is organized as follows. In section 2, the proposed discriminative features are described. In section 3, the experimental results are presented. Section 4 concludes the paper.

\section{DISCRIMINATIVE FEATURES}

Figure 1 shows the framework of discriminative feature extraction. The MFCC vectors are expanded into a high dimensional space using $d$-order polynomial expansion in order to have a better discrimination. Then, a set of independent SVM classifiers, each of which corresponds to a phone class, are trained to produce the projection functions, $\left\{f_{1}(),. f_{2}(),. \ldots, f_{\mathrm{C}}().\right\}$. The $C$ independent SVMs are constructed in one phone versus the rest manner so that each of the resulting feature dimensions can provide effective information to separate each phone from the others.

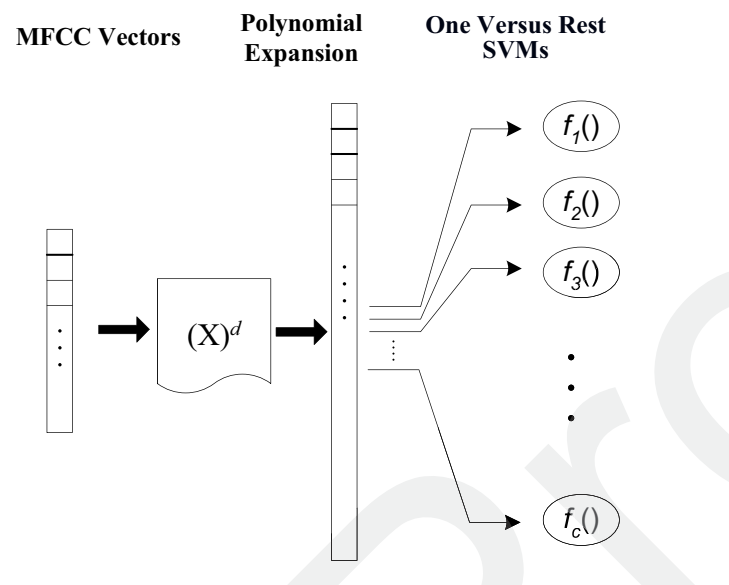

Figure 1: Discriminative feature extraction

One of the strategies for more discriminative acoustic features is to map the feature vectors of the original space into a new feature space by linear or nonlinear transformation, to have higher discriminative capability. In this way, we may use the polynomial expansion to expand the original MFCC vectors into a high dimensional space so that larger distances among the high dimensional feature vectors can be produced.

The generated vectors using polynomial expansion have a high dimensionality. In order to fit the discriminative features in the hidden Markov model structure of speech recognition, the high dimensional feature vectors need to be further projected into a low dimensional feature space. Dimensionality reduction is typically achieved through the linear combinations of samples in their original space. Linear combinations are particularly attractive because they are simple to compute and analytically tractable.

One way to reduce the feature dimension is to use Principle Component Analysis (PCA) [11] technique. PCA effectively reduces the dimension by finding the low dimensional subspace that best represents the full data with respect to a minimum squared error by keeping only the higher eigen values of the scatter matrix. Although PCA finds subspaces that are useful for representing the original high dimensional vector space, there is no guarantee that the resulting projection will be useful for discrimination between the samples of different classes. On the other hand, LDA [12] attempts to find an optimal linear transformation of input feature vectors such that the class separability in the new space is maximized.

As a variant, SVM has shown to be effective in separating high dimensional vectors in 2-class problems [13], in which SVM effectively projects the high dimensional vector $\mathbf{x}^{\prime}$ into a scalar value $f\left(\mathbf{x}^{\prime}\right)$. The classification of some known point in the input space $\mathbf{x}_{i}$ is $\mathrm{y}_{i}$ which is defined to be either -1 or +1 . If $\mathbf{x}^{\prime}$ is a point in the input space with unknown class, then

$$
\mathrm{y}^{\prime}=\operatorname{Sign}\left(\sum_{i=1}^{N} \alpha_{i} \mathrm{y}_{i} K\left(\mathbf{x}_{i}, \mathbf{x}^{\prime}\right)+d\right)
$$

where $\sum_{i=1}^{N} \alpha_{i} \mathbf{y}_{i}=0,0<\alpha_{i}, \mathrm{y}_{i}=\{-1,1\}, \mathrm{y}^{\prime}$ is the predicted class of point $\mathbf{x}^{\prime}$, the function $K($.$) is the kernel, N$ is the number of support vectors, $\alpha_{i}$ are adjustable weights and $d$ is a bias, both to be learned during training.

Suppose, we want to project a high dimensional vector to a lower dimensional space while preserving discrimination between different classes. This can be done using SVMs output scores. In this case, we consider the $f\left(\mathbf{x}^{\prime}\right)$ (i.e. the score) instead of classification decision:

$$
f\left(\mathbf{x}^{\prime}\right)=\sum_{i=1}^{N} \alpha_{i} \mathrm{y}_{i} K\left(\mathbf{x}_{i}, \mathbf{x}^{\prime}\right)+d
$$

where $f\left(\mathbf{x}^{\prime}\right)$ can be interpreted as the similarity between the SVM hyperplane and the input vector $\mathbf{x}^{\prime}$. In this way, the projection function is constructed by $C \mathrm{SVM}$ decision hyperplanes each of which trained to separate a specific target phone from its competing phone set. A natural way to construct these $C$ independent SVMs is to train them each deciding a target phone versus the rest. To do so, we denote high dimensional feature vectors labeled with the target phone as the positive set, and the rest as the negative set.

The $C$ SVM outputs form a reduced dimensional space, which is also known as output coding [14]. The code size $C$ of the defined output code vector is equal to the number of phones. Output coding, also known as error-correcting output coding, is a general method for solving multiclass problems by reducing them to multiple binary classification problems. In this paper, the continuous coding projection function is implemented in the way shown in Figure 2. To have better illustration, the simple case of 2-dimensional input vectors is considered.

After training a set of $C$ independent SVM classifiers $\left\{f_{1}(),. f_{2}(),. \ldots, f_{\mathrm{C}}().\right\}$, we project each high dimensional input vector $\mathbf{x}^{\prime}$ to a vector of $C$ real-valued $\mathrm{SVM}$ outputs $\mathbf{y}=\left\{f_{1}\left(\mathbf{x}^{\prime}\right), f_{2}\left(\mathbf{x}^{\prime}\right), \ldots, f_{\mathrm{C}}\left(\mathbf{x}^{\prime}\right)\right\}$ which is interpreted as discriminative features for subsequent phone recognition problem. The output coding $\mathbf{y}$ can be 
seen as a linear transformation of $\mathbf{x}^{\prime}$. Instead of original MFCC frames, the resulting output coding feature vectors are then fed into an HMM base-line phone recognizer to train the HMM acoustic models.

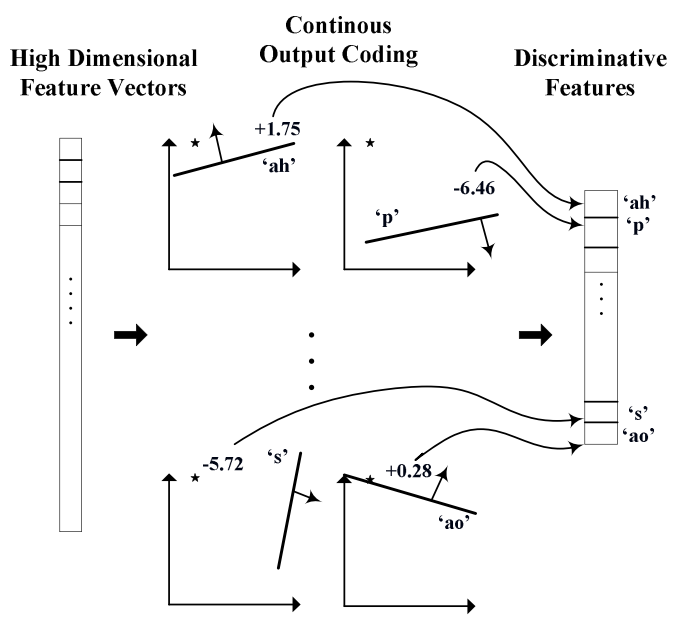

Figure 2: Continuous output coding process

\section{EXPERIMENTS}

In the experiments, we will evaluate the discriminating capability of the high dimensional feature vector based on the polynomial expansion of MFCC vectors. The evaluation is conducted by the framewise phone classification with the high-quality manual phone labeling in TIMIT corpus [15]. We will also evaluate the effectiveness of discriminative vectors based on the output coding method on the standard TIMIT speech recognition task.

\subsection{Experiment setup}

The training data set includes the 4,620 utterances from the standard 462-speaker training set in which 142,910 phone segments have been labeled. The test data set includes 192 utterances from the standard 24-speaker test set in which 7,215 phone segments have been labeled. We followed the HMM training practice in [16] for 48 phones and then mapping down to 39 phones for scoring purposes.

For each speech frame, a 39-dimensional feature vector is extracted, consisting of 12 MFCCs and normalized energy, plus their first and second order derivatives. Sentence-based cepstral mean subtraction was applied to acoustic normalization both in the train and test data.

For the speech recognition system, decision-tree based state-tying context-dependent triphone models had been used for the acoustic modeling. Approximately 1200 tied-states each having 16 mixture components have been built. A unigram phone language model, which was estimated using TIMIT dictionary, was applied to the phone recognition. HTK toolkit [17] was used for both acoustic model training and phone recognition.

\subsection{Discriminative feature vector}

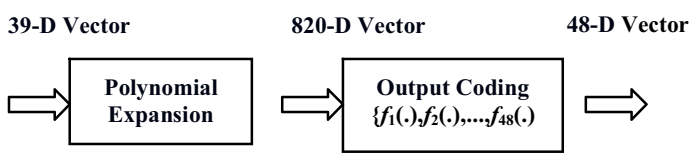

Figure 3: Discriminative feature vector extraction paradigm

Figure 3 shows the data flow of the discriminative feature vector extraction. The 39-dimension MFCC vectors were expanded into high dimensional space by calculating all the monomials up to order 2, resulting $\left(\begin{array}{c}(39+1)+2-1 \\ 2\end{array}\right)=820$ elements of feature vector. We expect that this high dimensional feature vector will have more discriminative capability to differ speech frames of one phone from another.

The obtained 820-dimension feature vectors were used to train the $48 \mathrm{SVMs}$ for discriminative feature vector based on the output coding projection. Each SVM was trained in the one phone versus the rest manner, using the high dimensional feature vectors of a phone as the positive set and the vectors from all other phones as the negative set. We thus obtain a discriminative vector with the dimension of 48 which is equal to the number of phones.

For the SVM training, the high quality manually time-aligned phone labels of TIMIT corpus were used with the SVMTorch toolkit [18]. There are about 1.1 million labeled frames in the TIMIT training data set. In order to train the SVMs efficiently, the training vectors in each of the phone classes were quantized up to 5000 centroids using k-means clustering algorithm.

\subsection{Framewise Phone Classification}

Figure 4 shows the framewise classification comparison among the original 39-dimension MFCCs, the 820dimension expanded feature vector based on polynomial expansion, and the 48-dimension discriminative feature vector based on the output coding technique. The target of this framewise classification experiment is to examine the discriminative capability of different feature vectors in a simple framewise phone classification task. It is shown that with enough training samples per phone class, the high dimensional feature vector outperforms the original MFCCs due to the larger distances among these feature vectors.

By projecting the high dimensional feature vector into a much lower feature space of 48 dimensions using the output codes of 48 SVMs with each SVM providing the discriminative information in the phone classification, we successfully reduced the high dimensional feature vector to a much lower feature space and further improved the discriminative capability at the same time. 


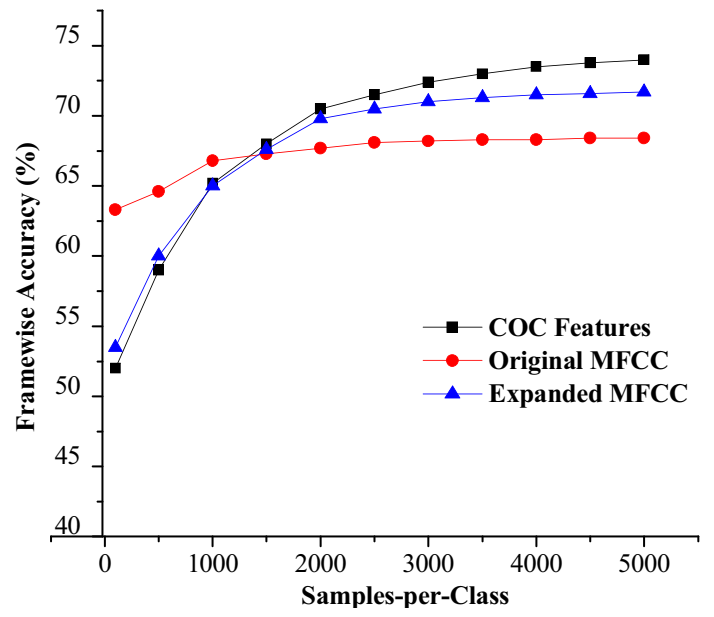

Figure 4: Comparison of the framewise phone classification accuracy among the original MFCC, polynomial expanded $M F C C$ and discriminative feature vector

\subsection{Phone recognition}

Table 1. Performance comparison of phone recognition between original MFCCs and discriminative feature vectors

\begin{tabular}{lc}
\hline Phone Recognition & Accuracy \\
\hline Original MFCC features & $70.24 \%$ \\
SVM-based discriminative features & $72.18 \%$ \\
\hline
\end{tabular}

Now the generated discriminative feature vectors are in low dimensions and can be easily fitted in the common adopted HMM framework. Table 1 compares the phone recognition performance using original 39-dimenson MFCCs and generated 48-dimension discriminative feature vectors.

The results show that the discriminative feature vector outperforms MFCCs in phone recognition accuracy on TIMIT task. The improvement in recognition result reflects the effectiveness of using the proposed framework for the discriminative features in speech recognition system.

\section{CONCLUSIONS}

In this paper, a new approach to generate discriminative acoustic features for speech recognition was proposed. It was based on the fact that the commonly used MFCC feature vectors are not discriminative in nature. Hence, using polynomial expansion, the vectors are expanded in a highdimensional feature space to produce larger distances between the sample frames of different phone classes. In order to benefit from the high-dimensional features, output coding technique was applied to project the high-dimensional features to a much lower feature space, so that a conventionally-trained HMM based ASR system can be directly adopted. It is shown that the enhanced discriminative capability of highdimensional feature space has been improved in the dimension reduction.

\section{REFERENCES}

[1] L.R. Bahl, P.F. Brown, P.V. De Souza, and R.L. Merver, "Maximum mutual information estimation of HMM parameters for speech recognition", Proc. ICASSP, pp. 49-52, 1986.

[2] Y. Normandin, R. Cardin, and R. Demori, "Highperformance connected digit recognition using maximum mutual information estimation", IEEE Trans. Speech Audio Proc., pp. 299-311, 1994

[3] E. McDermott, and S. Katagiri, "Prototype-based minimum classification error/generalised probabilistic descent training for various speech units", Computer Speech and Language, pp. 351-368, 1994.

[4] B.H. Juang, W. Chou, and C.H. Lee, "Minimum classification error rate methods for speech recognition", IEEE Trans. Speech and Audio Proc., pp. 257-265, 1997.

[5] D. Povey, and P.C. Woodland, "Minimum phone error and I-smoothing for improve discriminative training", Proc. ICASSP, pp. 105-108, 2002.

[6] F. Sha, and L.K. Saul, "Large margin hidden Markov models for automatic speech recognition", Advances in Neural Information Processing Systems, MIT Press, Cambridge, 2007.

[7] H. Jiang, X. Li, and C. Liu, "Large margin hidden Markov models for speech recognition", IEEE Trans. Audio, Speech and Language Proc., pp. 1584-1595, 2006.

[8] H. Bourlard, and N. Morgan, "Connectionist Speech Recognition:A Hybrid Approach". Kluwer Academic Publishers, Boston, 1994.

[9] H. Hermansky, D.P.W. Ellis, and S. Sharma, "Tandem Connectionist Feature Extraction for Conventional HMM Systems", Proc. ICASSP, 2000.

[10] S. Kajarekar, B. Yegnanarayana, and H. Hermansky, "A study of two dimensional linear discriminats for ASR," ICASSP, 2001.

[11] M. Kobayashi, and M. Aono, "Vector space models for search and cluster mining", Survey of Text Mining, in M.W. Berry, Springer, 2003.

[12] R.A. Fisher, "The Use of Multiple Measurements in Taxonomic Problems", Ann. Eugen. pp. 179-188, 1936.

[13] V. Vapnik, "The Nature of Statistical Learning Theory", Springer, N.Y., 1995.

[14] T.G. Dietterich, and G. bakiri, "Solving multi-class learning problems via error-correcting output codes", Journal of Artificial Intelligence Research, pp. 263-286, 1995.

[15] W.M. Fisher, G.R. Doddington, and K.M. GoudieMarshall, "The DARPA speech recognition research database: specifications and status", in Proc of DARPA Workshop on Speech Recognition, pp. 93-99, 1986.

[16] S. Young, "Large vocabulary continuous recognition: review", IEEE Signal Processing Magazine, pp. 45-57, 1996.

[17] S. Young, G. Evermann, D. Kershaw, J. Odell, D. Ollason, V. Valtchev, and P. Woodland, "The HTK Book (for HTK Version 3.2) ", Cambridge University Eng. Dept, 2002.

[18] R. Collobert, and S. Bengio, "SVMTorch: Support vector machines for large-scale regression problems", Journal of Machine Learning Research, pp. 143-160, 2001. 